\title{
Application of Scanning Electron Microscopy in the Characterization of Aschoff Nodules in Rheumatic Heart Disease
}

\author{
Prem Chopra, M.D.
}

\begin{abstract}
SUMMARY
Aschoff nodules (ANs) in excised atrial appendages from cases of rheumatic heart disease were examined with a scanning electron microscope (SEM). ANs could be identified with ease as nodular, fairly well defined cell aggregates lying in the subendocardium closely apposed to the myocardium. Cells comprising ANs were loosely packed in stacks. They were rectangular, oblong and angulated. The surface of some cells had fine ribbing while others were smooth having occasional blunt projections. SEM observations coupled with the findings of conventional and electron microscopy suggest that cclls composing ANs are of mesenchymal origin having characters of epithelioid cells, reticular cells and/or fibroblasts.
\end{abstract}

\section{Additional Indexing Words:}

Scanning electron microscope Aschoff nodule Rheumatic heart disease Epithelioid cell Reticular cell Fibroblast

\begin{abstract}
A $\mathrm{N}$ Aschoff nodule (AN) is a histologic marker of rheumatic heart disease (RHD). The classic human and experimental observations of Murphy ${ }^{1}$ suggested that the AN is of myogenic origin. This has been corroborated by other workers. ${ }^{2,3)}$ Ultrastructural analysis, however, revealed that Aschoff cells lack features of myofibres. ${ }^{41,5)}$ On the other hand, based on conventional and electron microscopy, ANs have been claimed to arise from a variety of mesenchymal cells other than myofibres. ${ }^{4)-8}$ )

This study was planned to evaluate the surface characteristics of cells comprising $\mathrm{AN}$ in an attempt to see if they have features of mesenchymal cells such as macrophages, which are favoured to be the cells of origin. To our knowledge, these are the first scanning electron microscope (SEM) observations of $\mathrm{AN}$.
\end{abstract}

From the All India Institute of Mcdical Sciences, New Delhi-110029, India.

Address for reprint: Prem Chopra, M.D., Department of Pathology, All India Institute of Medical Sciences, Ansari Nagar, New Delhi-110029, India.

Reccived for publication July 17, 1984. 


\section{Materials and Methods}

Left atrial appendages (LAA) excised from patients with RHD were evaluated. Six LAA which had numerous ANs and 4 samples that did not have any ANs in the subendocardium were studied in detail. In all cases except one, tissue was removed from a paraffin block and processed for SEM after thorough deparaffinization. One case which had several AN in the endocardium on cryostat section was processed fresh i.e. without prior fixation in formalin.

After deparaffinization, the area of the LAA bearing AN was appropriately trimmed to measure $0.8-1 \mathrm{~cm}$ square and $0.2-0.3 \mathrm{~cm}$ in thickness. Fresh tissue was fixed in $2.5 \%$ glutaraldehyde for about 24 hours. The specimens were washed thoroughly in $0.1 \mathrm{M}$ phosphate buffer in 3-4 washes of 2 hours each. Dehydration was carried out through increasing concentrations of acetone. Critical point drying was done using liquid $\mathrm{CO}_{2}$. The specimens were mounted on aluminium stubs with conducting paint. These were then coated with silver under vacuum in an atmosphere of argon gas. The specimens were screened in P-SEM-501B.

\section{RESULTS}

Using conventional light microscopy, numerous ANs were present within

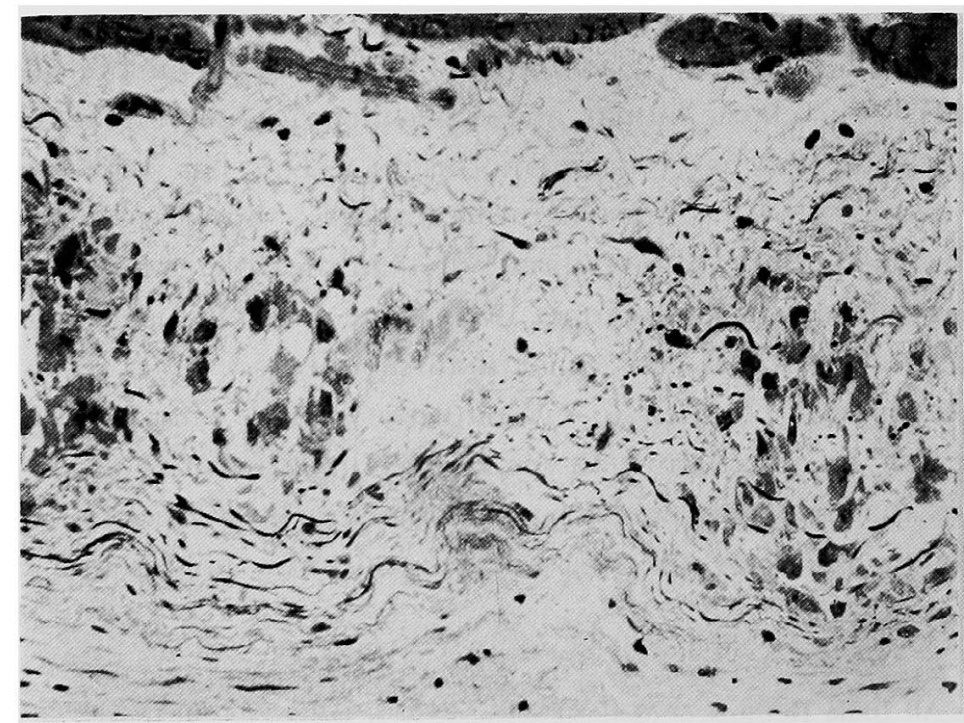

Fig. 1. Photomicrograph of Aschoff nodules (ANs) in the subendocardium. Myofibres can be seen in the upper part of the picture. Thick section, Toluidine blue, $\times 90$. 
the loose fibroconnective tissue of the subendocardium in all the 6 cases. These well formed (granulomatous phase) ANs were closely apposed to the myocardium (Fig. 1). The subendocardium of the 4 cases devoid of ANs, showed only loose fibroconnective tissue with occasional mesenchymal cells.

Ultrastructural examination of ANs revealed uni-, bi- and multinucleate groups of Anitschkow cells (AC) in the subendocardium. ${ }^{91}$ Cell membranes exhibited mild to moderate ruffing. The prominent rough endoplasmic reticulum (RER) often had dilated cisterns containing homogenous material. A variable number of mitochondria, some showing degenerative changes, were admixed with the RER. Abundant ribosomes were seen. Smooth surfaced vesicles beneath the cell surface and within the cytoplasm were striking in some cells. Only a few cells contained dense bodies.

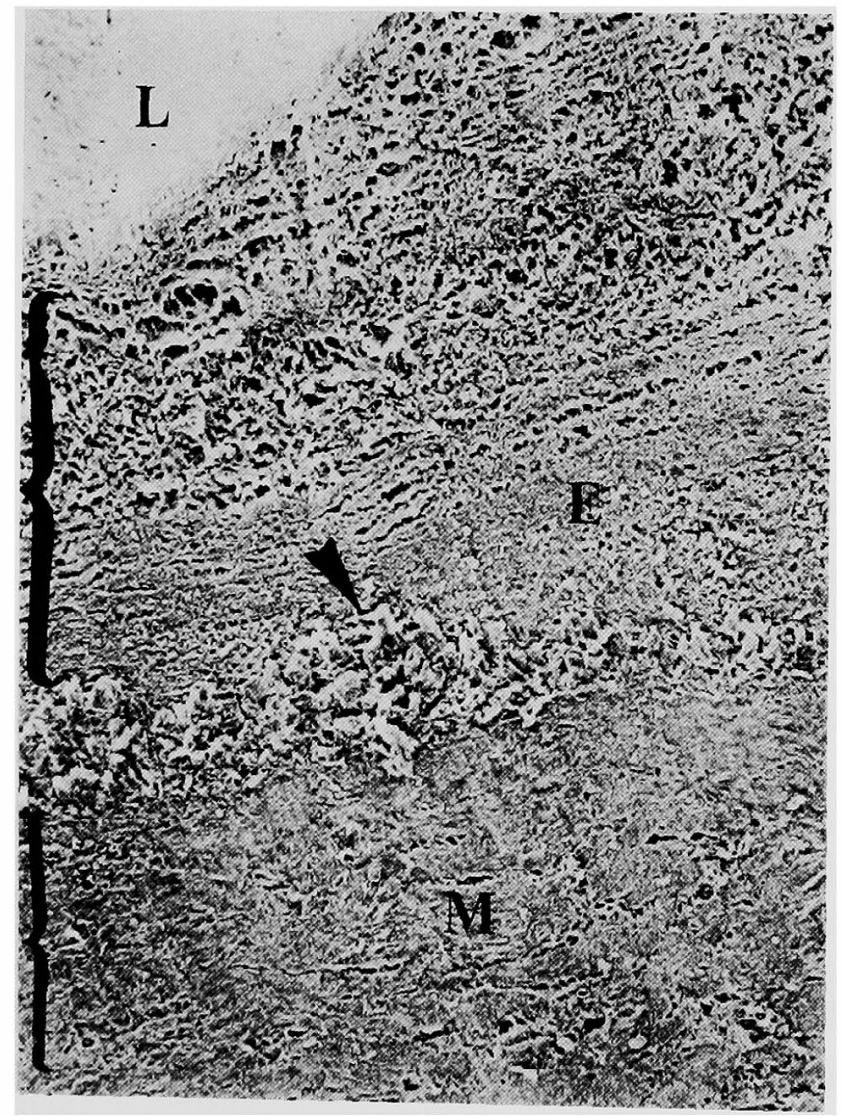

Fig. 2. Scanning electron micrograph (SEM) from a case of rheumatic heart disease (RHD). Numerous ANs were observed in the excised left atrial appendage (LAA). The ANs are irregular, nodular cell groups (arrow head) sandwiched between the endocardium and myocardium. The endocardium is markedly thickened $(\times 160)$. L=lumen; $\mathrm{E}=$ endocardium; $\mathrm{M}=$ myocardium. Larger bracket: endocardium, smaller bracket: myocardium. 


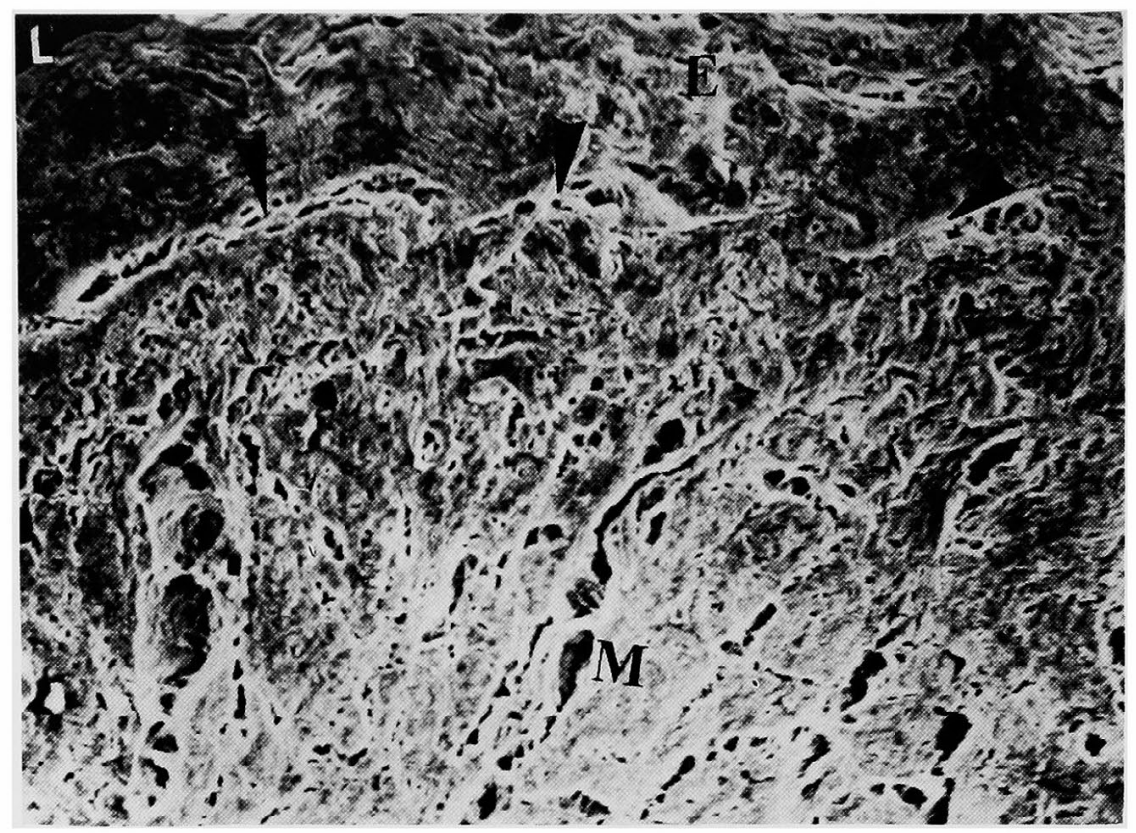

Fig. 3. Electron micrograph from a case of RHD where no ANs were encountered in the excised specimen. The endocardium $(E)$ is much thinner than that in Fig. 2. The junction between the endocardium and myocardium (M) is distinct (arrow heads). No ANs were encountered at the latter site in the entire specimen. Compare with Fig. $2(\times 160)$. L=lumen.

The ANs could be identified with ease with low power SEM. They were irregular, nodular, fairly well defined aggregates sandwiched between the deeper part of the endocardium and the myocardium (Fig. 2). This was in striking contrast to the cases where no ANs were encountered on light and scanning electron microscopy; the junction between the endocardium and myocardium was distinct (Fig. 3). Generally, thickening of the endocardium was more pronounced in cases where ANs were present (compare Figs. 2 and 3). On closer inspection, the cells within the ANs were rectangular, elongated, oblong and often angulated. They were closely packed and often stacked and bundled (Figs. 4-7). The surface of some cells had a finely reticulated appearance, while others were relatively smooth (Figs. 4-7). A few blunt villous projections were evident on the surface of some cells (Figs. $6,7)$.

\section{Discussion}

The surface characteristics of cells within $\mathrm{ANs}$ do not resemble macrophages, which have classical, numerous, villous projections that cover their 


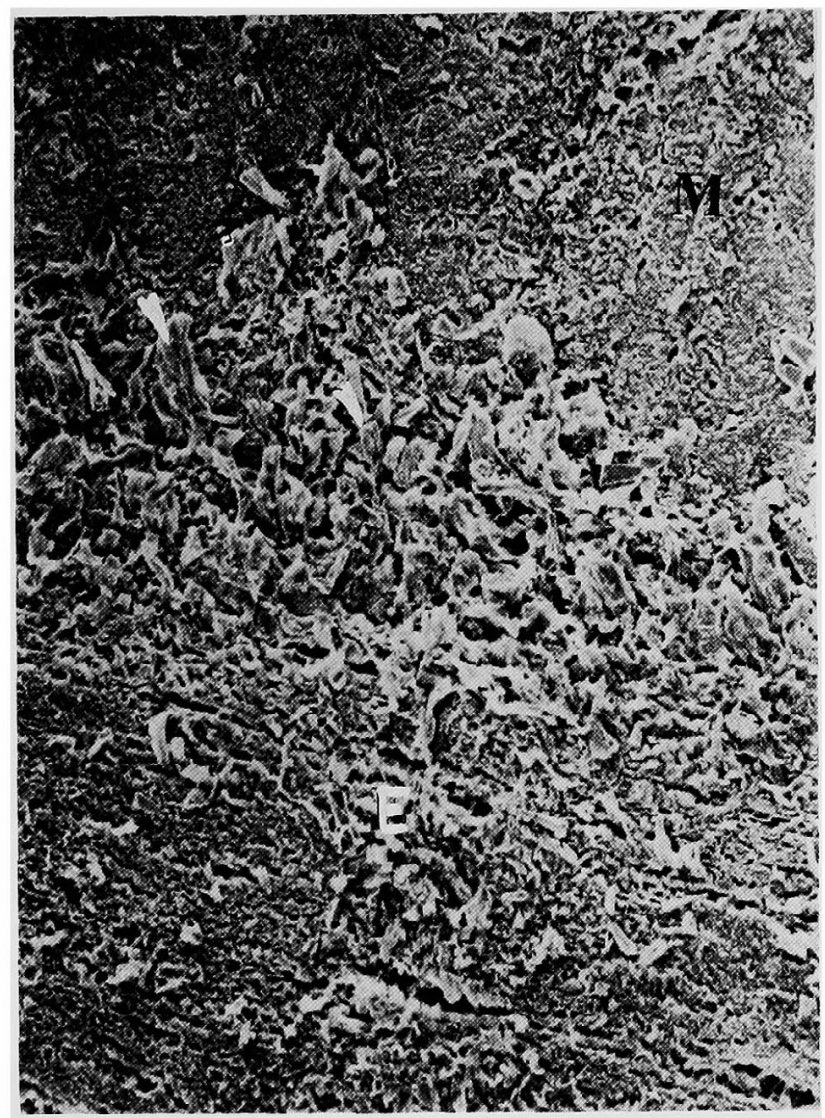

Fig. 4. Higher magnification view of Fig. 2 to show the AN lying between the endocardium (E) and myocardium (M). The cells are closely packed and stacked. The fine fibrillary appearance of the surface of several cells is evident (arrow heads) $\times 640$.

entire surface. ${ }^{10)}$ The AN cells had only occasional blunt knobs on their surface, which was either smooth or mildly reticulated. These cells do bear some resemblance to fibroblasts ${ }^{11)}$ and/or reticular cells. ${ }^{10), 12), 13)}$ A detailed account of surface appearance of normal and transforming fibroblasts has been given by Collard and Temmink. ${ }^{11)}$ Normal murine fibroblasts grow in vitro with widely extended lamellae and their surface is practically free of microvilli. However, their surfaces become adorned with microvilli at mitosis. ${ }^{11)}$ The AN cells appear to have some features of resting fibroblasts and bear a striking resemblance to reticular cells. ${ }^{10,12), 13 \text { ) }}$

Since $\mathrm{AC}$ have several ultrastructural features that resemble altcred macrophages (e.g., epithelioid cells ${ }^{91,14), 15)}$ and/or fibroblasts ${ }^{9)}$ and some characters of macrophages, ${ }^{4), 5}$ the SEM data further suggest that AC are of mesenchymal origin.4)-8) 


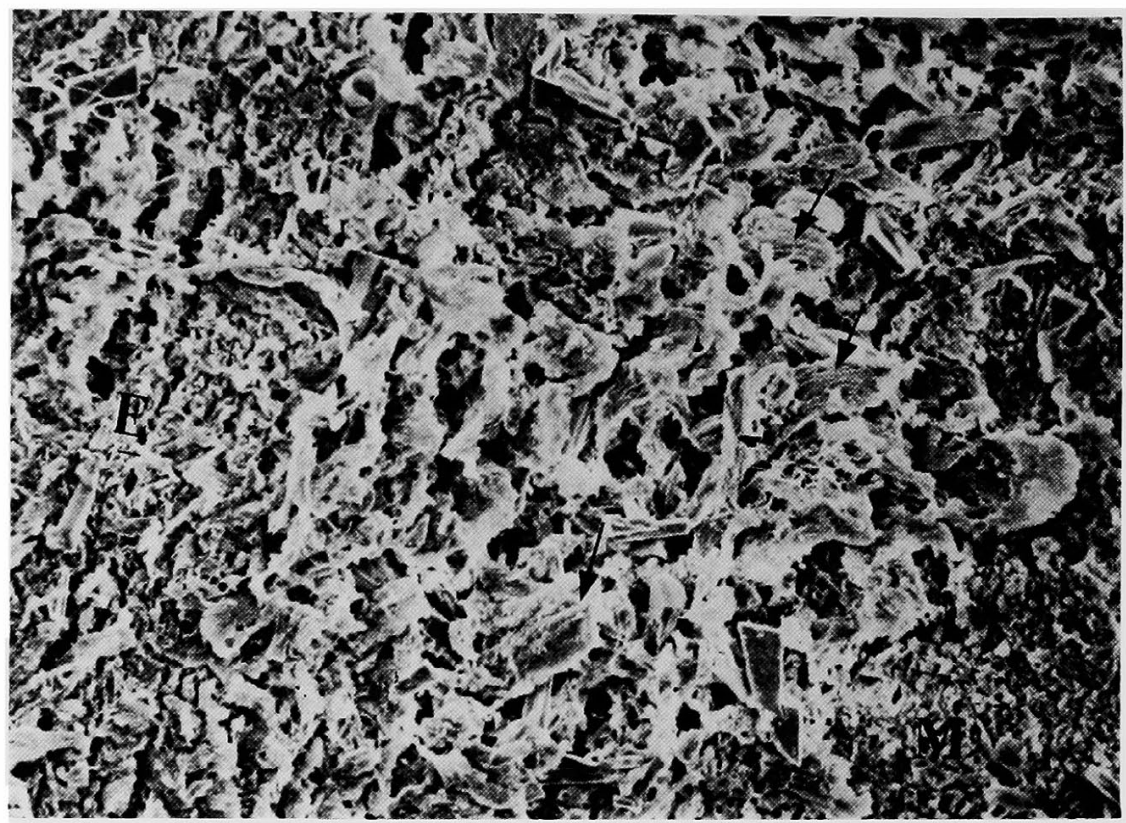

Fig. 5. Higher magnification view of Fig. 4 to show further details of cells within ANs. They are closely packed and are rectangular, oblong, angulated and elongated. Fine reticulations/ribbing of the surface is seen in many cells (arrows). Portions of myocardium (M) and endocardium (E) are also seen $(\times 1,250)$.

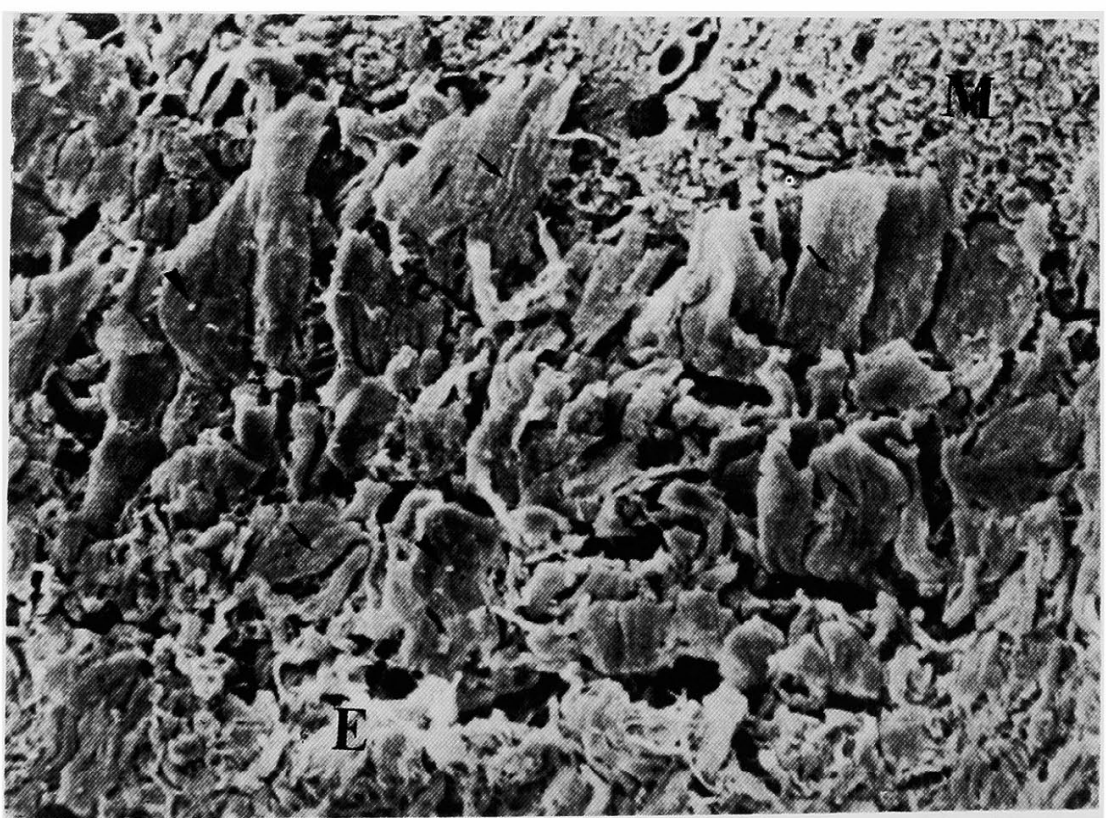

Fig. 6. SEM of another case that had numerous ANs in the LAA on conventional microscopy. Several cells have fine reticulations (small arrows) and blunt projections (small arrow heads) on their surface $(\times 1,250)$. 


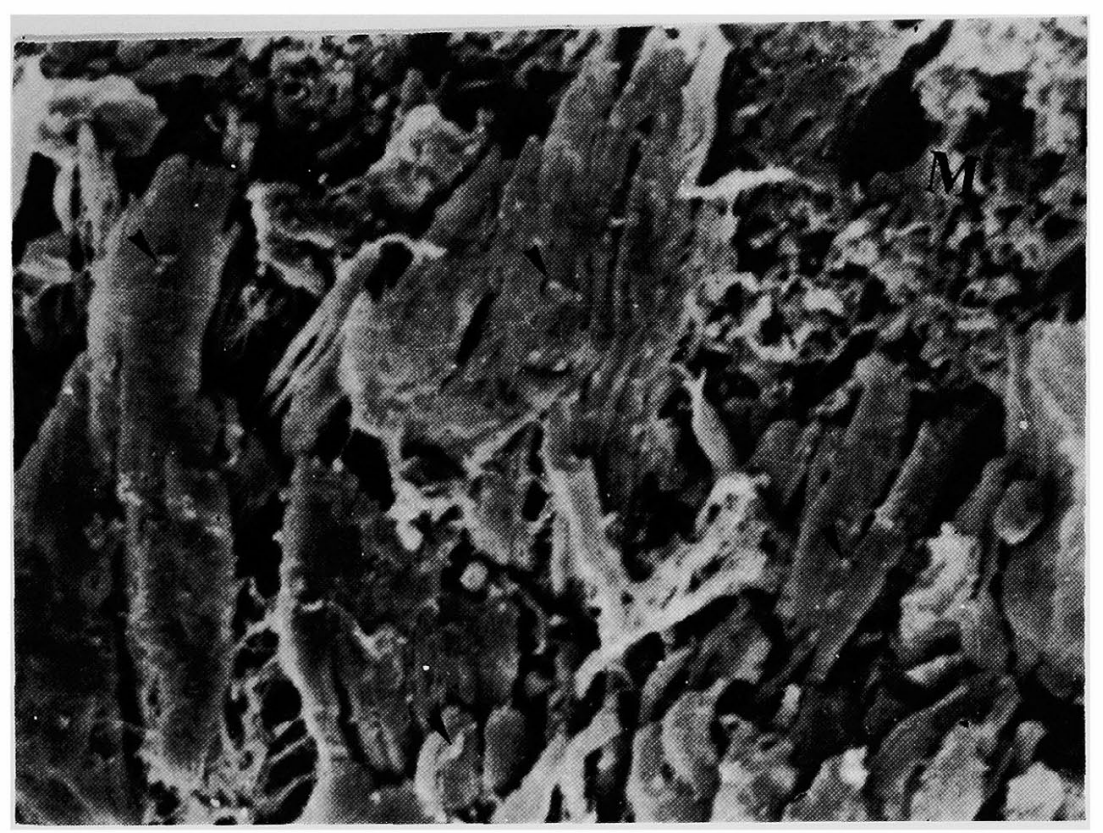

Fig. 7. Higher magnification view of Fig. 6 to show several blunt projections (small arrow heads) on the surface of cells comprising the AN. The ribbed appearance of cells is also evident. Part of the myocardium (M) is seen in the upper right hand corner of the picture $(\times 2,500)$.

Reticular tissue is a primitive connective tissue that consists of reticular cells, endothelial cells and macrophages. Reticular cells are believed to have potentialities of primitive mesenchymal cells, which can differentiate into phagocytic and fibroblastic forms. In addition they can give rise to macrophages and to early precursors of erythrocytes, leukocytes and perhaps to other cell types. ${ }^{12), 13)}$ The SEM characters of reticular cells have a number of features that we have observed in AC: they are rectangular or oblong and have a fine reticulated or ribbed surface. Villous projections characteristic of macrophages were lacking.

SEM observations have been reported in acute rheumatic valvulitis ${ }^{16)}$ and in diseased and normal human valves. ${ }^{17)}$ No reference, however, has been made to the appearance of $\mathrm{AC}$ or $\mathrm{AN}$ in these reports. To our knowledge, there are no previous reports concerning the morphology of $\mathrm{AC} / \mathrm{AN}$ in rheumatic heart disease.

\section{Aaknowledgments}

The author wishes to thank the regional facility in Department of Anatomy, All India Institute of Medical Sciences for help with scanning electron microscopy. 


\section{References}

1. Murphy GE: The characteristic rheumatic lesions of striated and of non striated or smooth muscle cells of the heart. Genesis of the lesions known as Aschoff bodies and those myogenic components known as Aschoff cells or Anitschkow cells or myocytes. Medicine 42: 73, 1963

2. Becker CG, Murphy GE: Demonstration of contractile protein in endothelium and cells of the heart valves, endocardium, intima, arteriosclerotic plaques and Aschoff bodies of rheumatic heart disease.

3. Mikat KW: Anitschkow cell development in rat myocardium. Arch Pathol 77: 47, 1964

4. Wagner BM, Siew S: Studies in rheumatic fever. V. Significance of the human Anitschkow cell. Human Pathol 1: 45, 1970

5. Lannigan R, Zaki SA: Ultrastructure of lesions of rheumatic carditis. J Pathol and Bacteriol $97: 449,1967$

6. Pienaar JG, Price HM: Ultrastructure and origin of Anitschkow cell. Am J Pathol 51 : 1963, 1967

7. Hutchins GM, Payne KT: Possible origin of myocardial Aschoff bodies of rheumatic fever from nerves. J Hopkins Med J 132: 315, 1973

8. Costero I, Barroso Mognel R, Chovez A, Monroy G, Contreras R: Rheumatic lesions in patients treated with cortisone. I. With special reference to endomyocarditis. Arch Inst Cardiol Mex 282: 155, 1958

9. Chopra P: Origin of Aschoff nodule. An ultrastructural, light microscopic and histochemical evaluation. Jpn Heart J 26: 227, 1985

10. Kessel RC, Kardon HH: 'Iissues and Organs, A lext Atlas of Scanning Electron Microscopy, WH Freeman \& Co, San Francisco, p 12, 13, 1979

11. Collard JG, Temmink JHM: Surface morphology and agglutinability with concanavalin A in normal and transformed murine fibroblasts. J Cell Biol 68: 101, 1976

12. Fujita $\mathrm{T}$ : Three dimensional microanatomy of reticular tissues. in Three Dimensional Microanatomy of Cells and Tissue Surfaces, ed by Didis LIA, Metta PM, Allen DJ, Elsevier, North Holland, p 89, 1981

13. Leeson TS, Leeson CK: Connective tissue proper. Chapter 3. in Histology, WB Saunders, Philadelphia, London, Toronto, p 130, 1981

14. Turk TL, Badenoch JP, Parker D: Ultrastructural observation on epithelioid cells of granulomas induced by Zirconium in the guinea pig. I Pathol 124: 45, 1978

15. Williams GT: Isolated epithelioid cells from disaggregated BCG granulomas. J Pathol 136: 1,1982

16. Siew S: The application of scanning electron microscopy in the investigation of acute rheumatic valvulitis. Human Pathol 11: 72, 1980

17. Harasaki H, Hanano J, Tanaka J, Tokunaga K, Torisu M: Surface structure of human cardiac valves: a comparative study of normal and diseased valves. J Cardiovasc Surg 19: 281,1978 